\title{
BIPLANE PHASED ARRAY FOR ULTRASONIC MEDICAL IMAGING
}

\author{
A. Shaulov, B. M. Singer, W. A. Smith†, and D. Dorman \\ Philips Laboratories, North American Philips Corporation \\ Briarcliff Manor, New York 10510 \\ $\dagger$ Present address: Materials Division, Code 1131 \\ Office of Naval Research, Arlington, Virginia 22217-5000
}

\begin{abstract}
We report a transducer structure that combines two orthogonal phased arrays which enable the real-time scanning of two orthogonal sectors. This biplane phased array is formed by partially dicing the opposite faces of a composite piezoelectric plate in orthogonal directions. Individual elements of the orthogonal arrays show broad radiation patterns close to the theoretical expectations for isolated elements. Arrays formed by patterning the electrode alone, without cross-dicing, show a narrower directivity pattern for a single element. This narrowing is a refractive effect due to the relatively high acoustic velocities in the composite material as compared to the sound velocity in the propagation medium. The concept of the biplane phased array forms the basis for a new class of transducer systems which combine two transducer arrays in a single piezocomposite plate.
\end{abstract}

\section{Introduction}

Advanced ultrasonic sector scanners employ phased array transducers to accomplish electronic steering and focusing of the acoustic beam [1-5]. These arrays are commonly fabricated from a piezoceramic plate by cutting it into narrow plank-shaped elements. In order to obtain a wide angular response free of grating lobes, the center-to-center element spacing is approximately a half wave-length of sound in tissue at the center frequency $[4,5]$. The concept of a biplane phased array which combines two orthogonal phased arrays was disclosed in a patent by 't Hoen [6]. This patent describes two orthogonal arrays formed on a composite piezoelectric disk by patterning the electrodes on the opposite faces of the disk. The two orthogonal arrays can be operated sequentially to scan the ultrasonic beam in two orthogonal planes. One scan is obtained by connecting the back-face electrodes to ground and the front-face electrodes to the phasing circuit. The orthogonal scan is obtained by reversing the electrode connections. Snyder, Kisslo and von Ramm [7] have also advanced a system employing such an array for cardiac scanning, as simultaneous longitudinal and transverse images of the heart can provide quite useful information on cardiac dynamics.

A fundamental requirement for a phased array is a wide angular radiation pattern for each element of the array. In this paper we describe an improved design of a biplane phased array in which this requirement is fulfilled by each of the orthogonal arrays.

0090-5607/88/0000-0635 \$1.00 ㅇ 1988 IEEE

\section{Experiments}

An improved structure of a biplane phased array is shown in Figure 1. The two orthogonal arrays are formed by partially dicing the opposite faces of an electroded plate of a composite piezoelectric material. The latter consists of a grid of PZT ceramic rods oriented perpendicular to the plate face and held together by an epoxy matrix [8]. The array may be provided with a backing layer and a crossed-diced matching layer as shown in Figure 2.

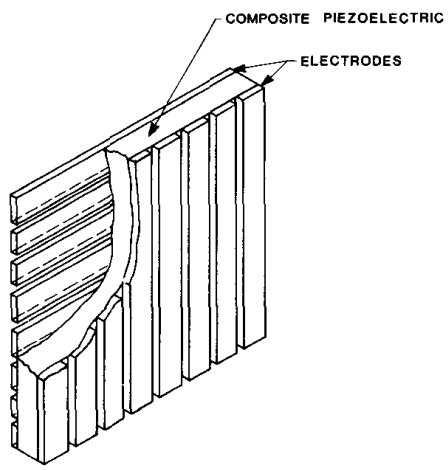

Figure 1: Biplane phased array formed by partially dicing the opposite faces of composite piezoelectric plate in orthogonal directions.

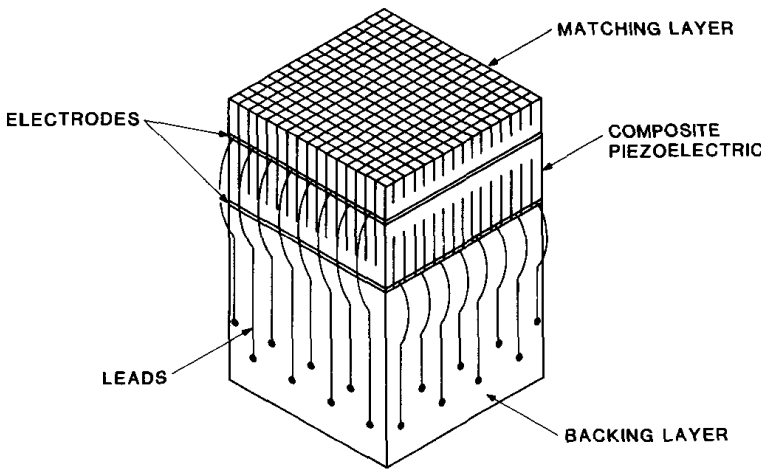

Figure 2: Biplane phased array with backing layer and crossed-diced matching layer.

1988 ULTRASONICS SYMPOSIUM -635 
Using this cross-dicing technique, prototype $3.2 \mathrm{MHz}$ biplane phased arrays were fabricated with a pitch of $0.25 \mathrm{~mm}$, so that each element (length $12 \mathrm{~mm}$ ) included two rows of PZT rods. Directivity measurements were performed in a water tank in a transmission mode using a single resonant pulse excitation. We present here experimental data for undiced and partially diced arrays to show the logical argument that leads to cross dicing the composites plates to form the biplane phased array.

Figure 3a shows the angular beam pattern for a single element in an undiced array. These data show a narrow beam pattern with dips near 38 degrees and peaks near 48 degrees, respectively. The diffraction theory for such a narrow radiator predicts a much broader beam pattern without sidelobes [9]. Further experiments with undiced array elements were performed using a different composite material made with a softer polymer (Spurr epoxy) Directivity measurements for a single element in this array showed a broader pattern without side lobes. However, the measured angular beam width was still much smaller than the theoretical expectation for an isolated radiator of the same dimensions.

Cross dicing experiments to improve the radiation pattern were performed using composite plates made with the stiffer epoxy. The two orthogonal arrays formed by patterning the electrodes were further accentuated by dicing the opposite faces of the plate to $30 \%$ of its thickness. A 12 micron Kapton foil served as a face plate to keep water from contacting the elements. The radiation profile from a single element in this array (Figure $3 \mathrm{~b}$ ) showed a beam width of 70 degrees at $-6 \mathrm{~dB}$ level which is $50 \%$ larger than that obtained with an undiced element.

Further improvement was obtained by cross dicing the elements to $60 \%$ of the plate thickness. In this array, directivity measurements were performed for elements belonging to each of the orthogonal arrays.

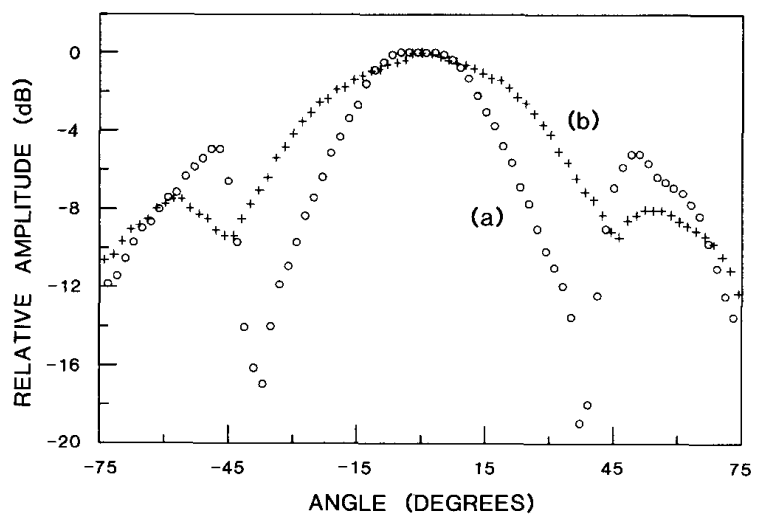

Figure 3: Radiation pattern from single element in composite biplane array formed by the electrode pattern alone (a) and by cross dicing the composite plate to $30 \%$ of its thickness (b).

\section{6 - 1988 ULTRASONICS SYMPOSIUM}

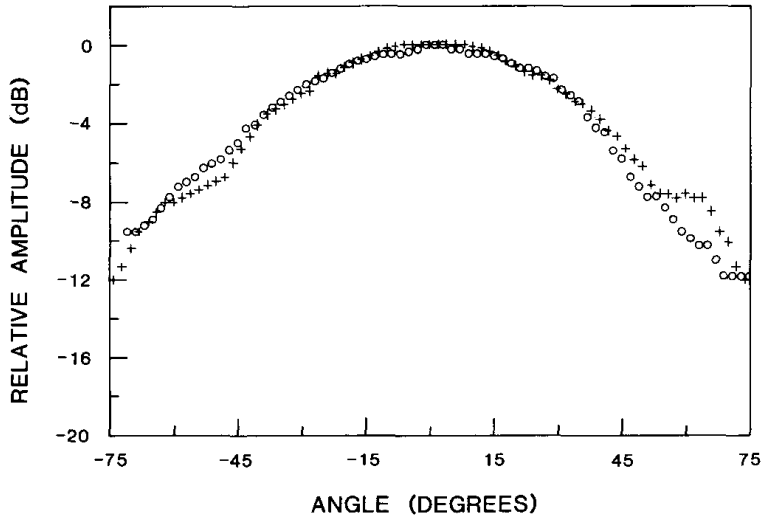

Figure 4: Radiation patterns from orthogonal elements in biplane phased array formed by cross dicing a composite plate to $60 \%$ of its thickness.

While exciting an element in the vertical array the horizontal electrodes on the opposite face were connected to ground. In a similar way, the vertical electrodes were grounded while exciting an element in the horizontal array. The circles and crosses in Figure 4 show the radiation patterns obtained from individual elements of the horizontal and vertical arrays, respectively. Both array elements show a broad radiation pattern with an angular width of 96 degrees at the $-6 \mathrm{~dB}$ level. This is close to the theoretical beam width of about 100 degrees expected for an isolated element of the same dimensions in a soft baffle [9].

\section{Discussion}

The experimental results indicate that the anomalies in the radiation pattern from an undiced phased array element are associated with the acoustic properties of the composite material. The combination of PZT rods and a polymer in a composite structure forms a highly anisotropic material. In common designs of such composites the acoustic velocities are higher than the speed of sound in water. This velocity mismatch creates refraction effects at the composite-water boundary which limit the angular width of the transmitted beam [10-12]. Considering only longitudinal waves in the composite, waves can be transmitted to water at a maximum angle corresponding to the critical angle $\theta_{c}$ measured from the normal to the plate:

$$
\theta_{c}=\sin ^{-1}\left(V_{w} / V_{c}\right),
$$

where $V_{w}$ is the speed of sound in water and $V_{c}$ is the speed of longitudinal waves propagating along the composite plate normal to the length of the ceramic rods. For the composite materials reported here we measured $V_{c}=2380 \mathrm{~m} / \mathrm{s}$. This gives a critical angle of 39 degrees which is near the location of the dips observed in the single element beam profile of the undiced array. 
The peaks at 48 degrees can be attributed to Lamb waves running in the composite plate in a direction perpendicular to the length of the elements [13]. These waves are generated as a result of mechanical stress gradients at the edges of the excited element. Phase matching at the water-composite interface implies that these waves radiate longitudinal waves into the water at an angle $\theta$ with the normal given by

$$
\theta=\sin ^{-1}\left(V_{w} / V_{L}\right)
$$

where $V_{L}$ is the speed of the Lamb waves. An estimate for $V_{L}$ in the composite array was obtained by measuring the time delay of the electrical signals generated on successive neighbors of the excited element as the wave passes through them. These measurements yielded $V_{L}=2160 \mathrm{~m} / \mathrm{s}$. The angle $\theta$ corresponding to this velocity is 44 degrees which is near the location of the side lobes observed in the radiation profile of an undiced element. The absence of side lobes in the composite made from Spurr epoxy is explained by the fact that the waves on the surface of this composite propagate at a velocity $V_{L}=1400 \mathrm{~m} / \mathrm{s}$ - lower than the speed of sound in water. Therefore such waves can not radiate into water.

The partial cross dicing of elements on opposite faces of the composite plate defines two orthogonal arrays with electrical elements divided into many mechanical subelements whose lateral dimensions are smaller than a wavelength (Figure 5). These small sub-elements radiate and receive acoustic energy at a wide angle because their lateral dimensions are insufficient for the wave phenomena of refraction to occur. The cross dicing also prevents narrowing of the beam due to cross talk between elements. The cross cuts confine the acoustic path between elements to a set of narrow strips that act as waveguides. The small transverse dimensions of these waveguides limit the number of propagating modes which they can support [14].

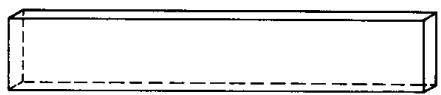

(a)

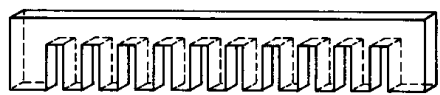

(b)

Figure 5: Transducer element (a) in a conventional phased array and (b) in the biplane phased array.
Moreover, cross dicing of the array elements improves the sensitivity of each element. This is because the vibration mode of each array element is changed from that of a width extensional mode (or "beam mode") of a plank to that of a length extensional mode of a set of bars. In the composite material reported here the electromechanical coupling factor of an array element increases from 0.59 to 0.65 after $60 \%$ dicing in orthogonal directions.

The concept of the biplane phased array forms the basis for a number of innovative transducers that can permit unique capabilities in ultrasound imaging. For example, a phased array can be combined with an annular array to allow high-resolution imaging and simultaneous volume flow measurements [15]. Another example is a combination of two orthogonal linear arrays [16]. Such a device would allow real-time scanning in a rectangular format of two orthogonal planes.

\section{Conclusions}

An improved biplane phased array is formed by cross dicing the elements on the opposite faces of a composite plate in orthogonal directions. The orthogonal arrays so formed exhibit a broad single-element directivity pattern. Composite piezoelectric materials and the cross dicing technique open new possibilities for the integration of other transducer systems into a single piezoelectric plate, e.g. crossed linear arrays and a combination of a phased array and an annular array. This new class of integrated transducer systems can add unique capabilities in ultrasound imaging.

\section{Acknowledgements}

We would like to thank Michael Athanas and John Zola of Philips Laboratories for their contribution in the fabrication of the composite materials and the transducer arrays. We are indebted to Prof. B. A. Auld of Stanford University for many useful discussions of array directivity phenomena. Thanks are also due to Drs. Pieter J. 't Hoen, S. Omar Ishrak, and E. James Pisa of Philips Medical Systems for valuable insights into transducer design and system requirements.

\section{References}

(1) H. E. Karrer and A. M. Dickey, "Ultrasound Imaging: An Overview", Hewlett-Packard Journal, Oct. 1983 , pp. 3 - 6.

(2) A. Macovski, "Ultrasonic Imaging Using arrays", Proc. IEEE 67, 484 (1979).

(3) J. F. Dias, "Construction and Performance of an Experimental Phased Array Acoustic Imaging Transducer", Ultrasonic Imaging 3, 352 (1981).

(4) G. S. Kino and C. S. DeSilets, "Design of Slotted Transducer Arrays With Matched Backing", Ultrasonic Imaging 1, 189 (1979). 
(5) J. F. Dias, "An Experimental Investigation of the Cross - Coupling Between Elements of an Acoustic Imaging Array Transducer", Ultrasonic Imaging 4, 44 (1982).

(6) P. J. 't Hoen, "Bi-Plane Phased Array for Ultrasound Medical Imaging", U. S. Patent 4,640,291, Feb. 3, 1987.

(7) J. E. Snyder, J. A. Kisslo and O. T. von Ramm, "Real-Time Orthogonal Mode Scanning of the Heart. I. System Design", Journal of the American College of Cardiology 4, 1279-1285 (1986).

(8) See the works cited in: W. A. Smith, "Composite Piezoelectric Materials for Medical Ultrasonic Imaging Transducers - A Review", Proceedings of the 1986 IEEE International Symposium on Applications of Ferroelectrics 249-255 (1986).

(9) A. R. Selfridge, G. S. Kino, and B. T. KhuriYakub, "A Theory for the Radiation Pattern of a Narrow-Strip Acoustic Transducer", Appl. Phys. Lett. 37,36 (1980).

(10) B. Noorbehesht, G. Flesher, and G. Wade, "Spatial Response of Arbitrarily Electroded Piezoelectric Plates by Plane-Wave Decomposition", Ultrasonic Imaging 2, 102 (1980).

(11) C. S. DeSilets, J. Fraser, and G. S. Kino, "Transducer Arrays Suitable for Acoustic Imaging", Proceedings of the 1975 IEEE Ultrasonics Symposium 148-152.

(12) B. A. Auld, C. DeSilets, and G. S. Kino, "A New Acoustic Array for Acoustic Imaging", Proceedings of the 1974 IEEE Ultrasonics Symposium 24-27.

(13) B. Delannoy, C. Bruneel, F. Haine, and R. Torguet, "Anomalous Behavior in the Radiation Pattern of Piezoelectric Transducers Induced by Parasitic Lamb Wave Generation", J. Appl. Phys. $51,3942(1980)$.

(14) B. A. Auld, Acoustic Fields and Waves in Solids, Chap. 10 (John Wiley and Sons, New York 1973).

(15) F. Hottier, P. Pesque, and R. Coursant, private communications.

(16) A. Shaulov, C. Hottinger, and M. Moshfeghi, private communications.

638 - 1988 ULTRASONICS SYMPOSIUM 